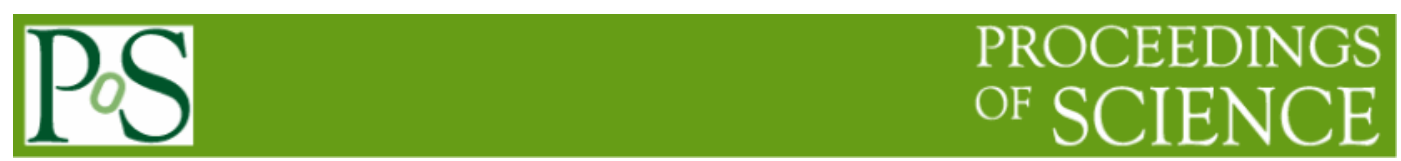

\title{
Future Neutrino Experiments
}

\author{
Peter Dornan ${ }^{1}$ \\ Imperial Cllege \\ Blackett Laboratory, Imperial College, London SW7 2AZ, UK \\ E-mail: P.Dornaneimperial.ac.uk
}

Precision neutrino physics offers a unique opportunity to investigate phenomena beyond the standard model. The talk reviews both accelerator and non-accelerator experiments to measure neutrino properties, which will take place in the near future and then describes new techniques for long baseline oscillation experiments which could be feasible for precision measurements in the next ten to twenty years.

Flavor Physics and CP Violation 2009

Lake Placid, NY, US,

May 27 - June 1, 2009,

\footnotetext{
$1 \quad$ Speaker
} 


\section{Introduction}

Neutrino properties are manifestly beyond the standard model but we know neither the cause nor the importance. It is at least now highly probable that the neutrino area will provide an additional source of $\mathrm{CP}$ violation and, should neutrinos be of Majorana origin, a possible explanation of the baryon asymmetry.

Over the last decade there have been major advances in quantifying the situation in the quark sector, albeit with no equivalent breakthrough in the understanding of the flavour problem. Hopefully, with a similar quantification in the neutrino and wider lepton sectors the desired advance will follow but this may well require precision experiments beyond the capabilities of those about to take data.

\section{What do we know?}

Current accepted experimental observations tell us

- There are three light active neutrino species (LEP/SLC)

- Mass of electron neutrino $<2.2 \mathrm{eV} / \mathrm{c}^{2}$ from the ${ }^{3} \mathrm{H}$ decay spectrum (Mainz[1], Troitsk[2])

- Muon Neutrinos produced in the upper atmosphere disappear (SuperK[3])

- Muon Neutrinos produced in $\pi$ decay disappear (K2K[4], MINOS[5])

- Electron Neutrinos produced in the sun disappear (Many experiments)

- The total flux of neutrinos from the sun stays constant (SNO[6])

- Electron antineutrinos from reactors disappear and then reappear (Kamland[7])

All these observations strongly favour a three family neutrino oscillation. There are two additional results, not universally accepted, but, if confirmed, would have profound consequences.

- The appearance of electron antineutrinos in a muon antineutrino beam (LSND[8]). If confirmed the conventional three neutrino scenario would be excluded as there would probably be one or more sterile neutrinos.

The observation that the decay ${ }^{76} \mathrm{Ge}{ }^{76} \mathrm{Se}+\mathrm{e}^{-}+\mathrm{e}^{-}$has a half life of $T_{1 / 2}^{0 v}=2.3_{-0.31}^{+0.44} \cdot 10^{25}$ years (H.V.Klapdor-Kleingrothaus et al[9]). If true then neutrinos are Majorana and the masses are $\sim 0.5 \mathrm{eV}$

\section{Oscillation Formulae}

Neutrino oscillation demonstrates that lepton flavour is not conserved and the neutrino mass eigenstates do not equal the flavour eigenstates. For a two state system this is parameterised with a unitary $2 \times 2$ matrix with one parameter, the mixing angle $\theta$

$$
\left(\begin{array}{l}
v_{e} \\
v_{\mu}
\end{array}\right)=\left(\begin{array}{cc}
\cos \theta & \sin \theta \\
-\sin \theta & \cos \theta
\end{array}\right)\left(\begin{array}{l}
v_{1} \\
v_{2}
\end{array}\right)
$$


which leads to an oscillation probability dependent on the mass difference of the two mass eigenstates through $\Delta m_{12}^{2}=m_{2}^{2}-m_{1}^{2}$

$$
\operatorname{Prob}\left(v_{\mathrm{e}} \rightarrow v_{\mu}\right)=\sin ^{2} 2 \theta \sin ^{2} \Delta_{12} \quad \text { with } \sin \Delta_{12}=\sin \left(\frac{\Delta m_{12}^{2} L}{E}\right)
$$

The oscillation rate depends upon the mixing angle, the magnitude of the difference of masses squared, the energy of the neutrinos and the distance they travel, the baseline, $L$. It is easy to see from this formula that degenerate solutions exist for different $\theta$ and $\Delta m^{2}$ but this can be removed by taking data at more then one L/E. In practice this is natural as long as the energy resolution is less than the energy spread of the beam.

For 3 generations there are 2 mass differences, 3 mixing angles and a phase, $\delta$, the Dirac phase, with the possibility of two additional phases should neutrinos be Majorana. The matrix is $\left(\begin{array}{l}v_{e} \\ v_{\mu} \\ v_{\tau}\end{array}\right)=\left(\begin{array}{ccc}1 & 0 & 0 \\ 0 & \cos \theta_{23} & \sin \theta_{23} \\ 0 & -\sin \theta_{23} & \cos \theta_{23}\end{array}\right)\left(\begin{array}{ccc}\cos \theta_{13} & 0 & \sin \theta_{13} e^{-i \delta} \\ 0 & 1 & 0 \\ -\sin \theta_{13} e^{-i \delta} & 0 & \cos \theta_{13}\end{array}\right)\left(\begin{array}{ccc}\cos \theta_{12} & \sin \theta_{12} & 0 \\ -\sin \theta_{12} & \cos \theta_{12} & 0 \\ 0 & 0 & 1\end{array}\right)\left(\begin{array}{ccc}e^{-i \alpha / 2} & 0 & 0 \\ 0 & e^{-i \beta / 2} & 0 \\ 0 & 0 & 1\end{array}\right)\left(\begin{array}{l}v_{1} \\ v_{2} \\ v_{3}\end{array}\right)$

and the oscillation is in general a function of the 3 three angles, the Dirac phase and the two squared mass differences through $\sin \Delta_{12}$ and $\sin \Delta_{13}$. Vacuum oscillation experiments cannot therefore determine the mass hierarchy. However, due to the different interaction rates the oscillation probabilities are altered as the neutrinos pass through matter and as a consequence

$$
\sin \Delta_{13} \Rightarrow \frac{\sin \left(\Delta_{13}-A L\right)}{\left(\Delta_{13}-A L\right)} \Delta_{13} \quad \text { and } \quad \sin \Delta_{12} \Rightarrow \frac{\sin (A L)}{A L} \Delta_{12}
$$

with $A=G_{F} N_{e} / \sqrt{2}$ the density factor. Thus the oscillation becomes sensitive to the sign of $\Delta m^{2}$ with the sensitivity depending upon $A L$, the density and the baseline

\section{Future Non-Oscillation Experiments}

\subsection{Determination of the Neutrino Mass}

Oscillations demonstrate that neutrinos are massive and dependent upon the modulus of the mass squared differences, $\left|\Delta m_{i j}^{2}\right|$, and in matter can be sensitive to the sign. However no information can be retrieved on the absolute mass. This is one of the most important and also one of the most difficult measurements to make.

Cosmological data can give bounds on $\sum m\left(v_{i}\right)$ but these are model dependent and there is not total agreement about the interpretation. A conservative current bound is $<\sim 0.7 \mathrm{eV}$ and there are hopes that this could improve within the next few years but the systematic uncertainty arising from the model dependence is likely to remain difficult to quantify. The rate of neutrinoless double beta decay $(0 v \beta \beta)$ depends on the quantity $m_{e e}=\left|\sum U_{e i}^{2} m_{i}\right|$ and thus the 
absolute mass, but this requires neutrinos to be Majorana and extracting an accurate value is complicated by uncertainties in the nuclear matrix elements.

\subsubsection{Direct mass determination}

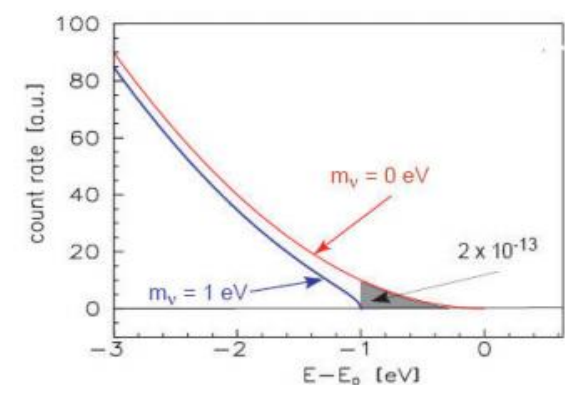

Fig 1. The endpoint of the ${ }^{3} \mathrm{H}$ beta spectrum dependent upon the neutrino mass

Currently the only procedure which can approach the sensitivity required to give a measure of the neutrino mass with minimal theoretical uncertainty is the measurement of the endpoint of the electron spectrum in beta decay. Tritium is the preferred element for this. It has a low $\mathrm{Q}$ value of $18.6 \mathrm{keV}$, a half life of $12.3 \mathrm{yr}$ and both it and the ${ }^{3} \mathrm{He}$ daughter have simple electron configurations. It is a super-allowed nuclear transition so there are no corrections from the nuclear matrix elements.

The sensitivity results from both the measurement of the maximum electron energy and the shape of the spectrum very close to the endpoint, shown in Fig 1. This shape is given by

$$
\frac{d N}{d E} \alpha F(Z, E) p E\left(E_{0}-E\right)\left[\left(E_{0}-E\right)^{2}-m_{v}^{2}\right]
$$

and thus the measurement yields the square of the 'electron neutrino' mass defined by $m^{2}\left(v_{e}\right)=\sum\left|U_{e i}\right|^{2} m_{i}^{2}$. In principle effects could be seen of the individual mass states however no experiment currently contemplated would have such a resolving power. Previous experiments at Mainz ${ }^{1}$ and Troitsk $^{2}$ have set upper limits around $2.2 \mathrm{eV}$ for $m\left(v_{e}\right)$ but within the next few years the KATRIN experiment at Karlsruhe (Fig 2)) has the goal of $\sim 0.2 \mathrm{eV}$. This is a major extrapolation on the earlier Mainz experiment and now, with the tritium source two spectrometers and the detector, is over $60 \mathrm{~m}$ in length. Data taking is now scheduled for 2012.

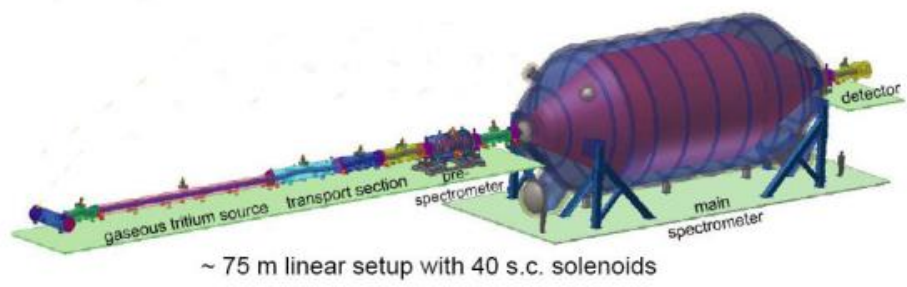

Fig 2, Schematic of the KATRIN Experiment

\subsubsection{Neutrinoless Double Beta Decay}

The most probable explanation for an observation of neutrinoless double beta decay is that it has proceeded via the diagram in Fig 3 which requires the exchanged neutrino to be massive and Majorana, i.e. for neutrino and antineutrino not to be distinct. At the present time this would appear to be the only method to prove the Majorana nature which is assumed in many theories for the low neutrino mass and the necessary rate of $\mathrm{CP}$ violation to explain the baryonantibaryon asymmetry via leptogenesis. It is thus of vital importance. 
The rate of $0 v \beta \beta$ is given by $\left(T_{1 / 2}\right)^{-1}=G^{0 v}\left|M_{G T}^{0 v}-M_{F}^{0 v}\right|^{2} m_{e e}^{2}$ with the unknowns the

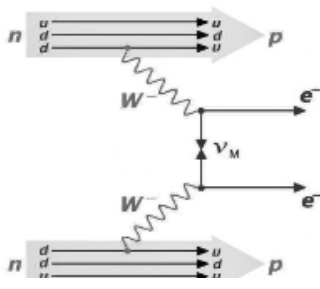

Fig 3. Neutrinoless double beta decay via the exchange of a light Majorana neutrino nuclear matrix elements and the quantity $m_{e e}=\left|\sum U_{e i}^{2} m_{i}\right|$. Thus a measurement of a double beta decay rate will confirm the Majorana nature of the neutrino and give a value for the absolute mass to the degree that the nuclear matrix elements are known. As stated above a positive result has been claimed, but it is certainly in need of confirmation. Excluding this measurement current limits yield lower limits on the $0 v \beta \beta$ half life in the region of $10^{23}$ to $10^{25}$ years corresponding to $\mathrm{m}_{\mathrm{ee}}$ values $\sim 1 \mathrm{eV}$.

The experiments focus on isotopes which undergo normal double beta decay and then look for a signal corresponding to the two beta electrons having an energy sum equal to the Q-value of the transition. The expected rates are very small, from 6 to 10 orders of magnitude lower than the two neutrino rate, and hence energy resolution and background elimination are critical. The experiments can be broadly split into two categories, purely calorimetric homogeneous ones where the signal is an energy pulse at the correct value and heterogeneous ones which also add tracking capabilities to identify the two electrons. Dominant backgrounds are from the end of the two neutrino beta decay spectrum and from contaminant radioactive decays, hence the need for high purity components and a low background environment. The tracking detectors have an advantage in eliminating the radioactive background but have poorer energy resolution and so the two neutrino decay becomes more serious.

There is presently a very lively programme of experiments in this area and some of these which aim for results during the next decade are:

- GERDA, Semiconductor calorimeter experiment using ${ }^{76} \mathrm{Ge}$ building upon the expertise from the original Heidelberg-Moscow and IGEX experiments. A first aim is to establish if the claimed observation of a signal by KlapdorKleingrothaus et al. is valid.

- CUORE. A cryogenic bolometric experiment using ${ }^{130} \mathrm{Te}$, evolving from CUORICINO.

- SuperNEMO. A tracking + calorimeter detector evolving from NEMO-3 which will concentrate on ${ }^{100} \mathrm{Mo}$ and ${ }^{150} \mathrm{Nd}$

- EXO-200. Employing a liquid Xe TPC this will use both ionisation and scintillation from ${ }^{136} \mathrm{Xe}$.

- Majorana. This will also use ${ }^{76} \mathrm{Ge}$ and it is assumed that if the next generation of investigations fail to see a signal it will

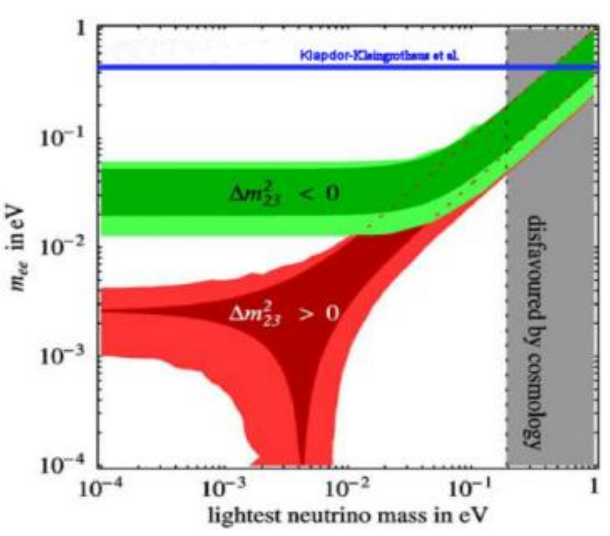

Fig 4. The value of $m_{e e}$ in terms of $m_{1}$. The green and red bands correspond to the inverted and normal hierarchies and the blue line to the claimed result from Klapdor-Kleingrothaus et al. (plot from the GERDA proposal) 
combine with GERDA for a multi tonne experiment.

- MOON. Investigating the use of tracking and calorimetry for ${ }^{100} \mathrm{Mo}$.

- COBRA. Prototyping the use of the semiconductor CdZnTe.

- SNO+. Planning to fill the SNO detector with liquid scintillator containing ${ }^{150} \mathrm{Nd}$. This will be significantly larger than other experiments although with less good energy resolution.

These experiments aim to reach half lives $>\sim 10^{27}$ years corresponding to values of $\mathrm{m}_{\mathrm{ee}}$ $<\sim 0.02 \mathrm{eV}$. Regrettably, even such sensitivities may not be adequate to determine if neutrinos are Majorana, and may just give an improved upper limit on the Majorana mass and no information at all if neutrinos are Dirac. This is more probable if the normal hierarchy of masses holds as can be seen from Fig 4. Nevertheless these experiments are vitally important and as systematics, particularly the backgrounds, are crucial for the sensitivity it is necessary that the experiments are conducted with different techniques and different nuclei.

\subsection{Near-future Oscillation Experiments}

\subsection{1 $\theta_{13}$}

The major goal of the forthcoming oscillation experiments is the determination of the unknown angle $\theta_{13}$. So far the best information is the upper limit on $\sin ^{2} 2 \theta_{13}$ of 0.19 set by the Chooz reactor experiment[10], although latest results form MINOS are beginning to contribute to the limit. Determination of $\theta_{13}$ is critical for the future direction of neutrino physics as it dictates the sensitivity of oscillation experiments to the $\mathrm{CP}$ violating phase $\delta$ and the mass hierarchy. In addition $\theta_{13}$ itself is an important parameter; if it is zero it presumably must indicate an unknown underlying symmetry, if not zero the value gives a measure of the breaking of tribimaximal mixing ${ }^{[}[11]$ - critical if tribimaximal mixing does indeed have a yet to be understood justification.

There are two current approaches for the determination of $\theta_{13}$ - accurate measurement of the disappearance of electron antineutrinos from a reactor and measurement of the appearance of electron neutrinos in a muon neutrino superbeam.

\subsubsection{Reactor Experiments}

Three reactor experiments are being constructed with the aim of starting data taking in the near future, Double Chooz in France, Daya Bay in China and RENO in Korea. Unlike the original Chooz experiment all three employ similar near and far detectors. Two main factors for the overall sensitivity are the neutrino rate, which is dependent on the power of the reactor, and the minimisation of systematic uncertainties which relies upon the near/far comparison. The disappearance probability is dominated by two oscillation terms

$$
\sin ^{2} 2 \theta_{13} \sin ^{2} \Delta_{13}+\cos ^{4} \theta_{13} \sin ^{2} 2 \theta_{12} \sin ^{2} \Delta_{12}
$$

As $\theta_{13}$ is known to be small the second, lower frequency, term dominates the oscillation except where $\Delta_{12}$ is close to zero and so $\theta_{13}$ is determined from the amplitude of the 


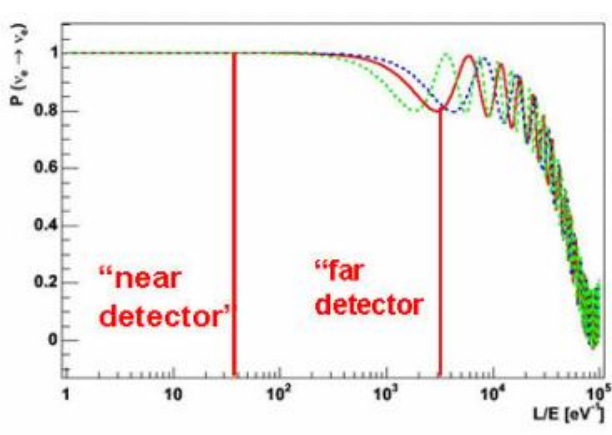

Fig 5. Illustration of primary and secondary oscillations for a reactor determination of $\theta_{13}$.

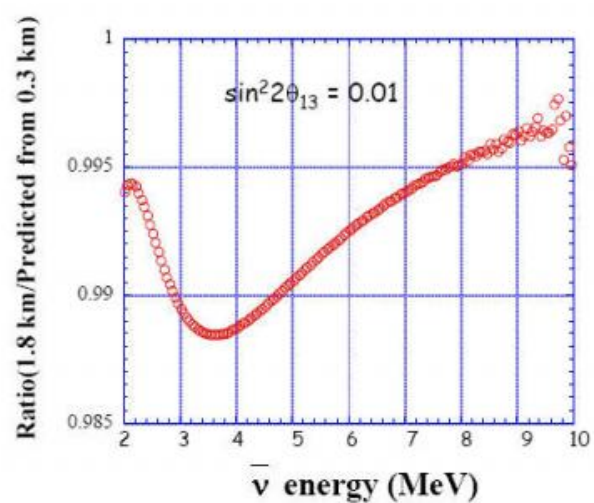

Fig 6. Predicted Daya Bay Near/Far Ratio for $\sin ^{2} 2 \theta_{13}=0.01$

secondary, higher frequency, oscillation at a short distance from the reactor. For illustration, the oscillation pattern is shown as a function of L/E in Fig 5 although in practice the signal will be smaller. In Fig 6 the far/near ratio for the Daya Bay experiment is shown for $\sin ^{2} 2 \theta_{13}=0.01$ showing that the effect is only $\sim 1 \%$ and hence the need for the experimental systematics to be well under control. An important advantage of reactor experiments for $\theta_{13}$ arises from the fact that there is no dependence on $\delta$ and the baselines are so low that matter effects are negligible.

Double Chooz is expected to be the first to start data taking in 2010 with the other two aiming for 2011. Should $\theta_{13}$ be zero or very small all experiments aim for an upper limit on $\sin ^{2} 2 \theta_{13}$ of 0.01 to 0.02 .

\subsubsection{Longbaseline Experiments}

LBL experiments are built around the standard method of producing a neutrino beam, protons hit a target producing pions, one sign of which is then focussed by one or more magnetic horns and then allowed to decay yielding a muon neutrino, or antineutrino beam depending on the pion sign. An irreducible background arises from kaons in the beam which have a $5 \% \pi^{0} e^{+} v_{e}$ decay mode, contaminating the beam with electron neutrinos.

Two experiments will commence data taking in the near future, T2K and Nova. They will be the first to use the off-axis technique whereby the far detector sits at a small angle off-axis giving a much smaller energy spread. The energy of the beam can thus be tuned by choosing the angle and optimised so that the far detector sits at the first oscillation maximum. The main goals of these experiments can be summarised as follows

- Measurement of $\theta_{13}$ by observing electron neutrino appearance.

- Improvement of the atmospheric parameters $\theta_{23}$ and $\Delta m_{13}^{2}$ from muon neutrino disappearance

- In the case of Nova, determination of the mass hierarchy should $\theta_{13}$ be sufficiently large

The dominant terms in the probability for the $v_{\mu}$ to $v_{\mathrm{e}}$ transition are

$$
\begin{aligned}
& \sin ^{2} 2 \theta_{13} \sin ^{2} \theta_{23} \sin ^{2} \Delta_{13}+\sin 2 \theta_{13} \cos \theta_{13} \sin 2 \theta_{23} \sin 2 \theta_{12} \sin \Delta_{13} \sin \Delta_{12} \cos \left(\Delta_{23}+K \delta\right) \\
& +\sin ^{2} 2 \theta_{12} \cos ^{2} \theta_{23} \cos ^{2} \theta_{13} \sin ^{2} \Delta_{12}
\end{aligned}
$$


with $K=+1$ for neutrinos and -1 for antineutrinos. As $\theta_{13}$ is known to be small the second term is the important one and it is therefore clear that an ambiguity exists as a result of the unknown $\delta$ which will not be resolved in these experiments as the off-axis technique means they effectively operate at a single $\mathrm{L} / \mathrm{E}$. Thus the unknown $\delta$ gives a limit to the accuracy with which $\theta_{13}$ can be determined. Both experiments claim sensitivity in the range 0.003 to 0.03 for $\sin ^{2} 2 \theta_{13}$ dependent on the values of $\delta$ and $\theta_{23}$. This effect is shown in Fig 7 which gives the expected sensitivity for $\mathrm{T} 2 \mathrm{~K}$.

The $\Delta$ quantities include the matter effects and if these are sufficiently large the mass hierarchy can be

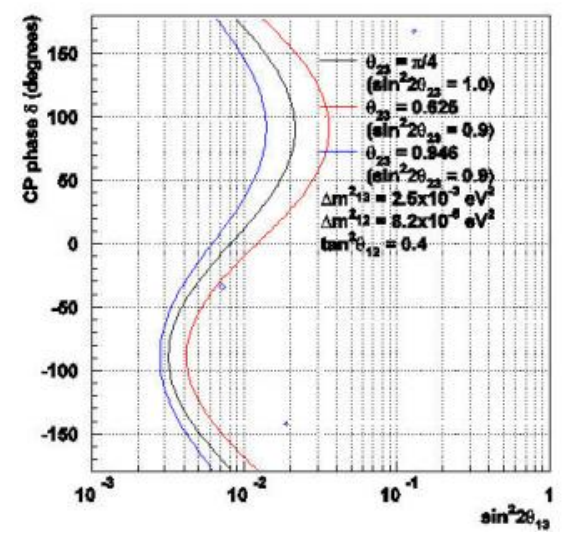

Fig 7. Expected $\sin ^{2} 2 \theta_{13}$ sensitivity for T2K dependent on the values of $\delta$ and $\theta_{23}$. determined from $\Delta_{13}$ in the first term. For T2K, with a baseline of $295 \mathrm{~km}$, the matter effects are below the sensitivity however this may not be the case for Nova with a baseline of $795 \mathrm{~km}$. However the sensitivity to the mass hierarchy is also dependent on the amplitude of the first term which involves $\sin ^{2} 2 \theta_{13}$ and consequently the ability of Nova to measure the mass hierarchy depends upon $\theta_{13}$. Both experiments are planning to take data until $\sim 2016$. and both have potential upgrade paths.

By that date it is conceivable that, if $\theta_{13}$ is large enough, by combining the reactor results, dependent purely on $\theta_{13}$, with T2K, dependent on $\theta_{13}$ and $\delta$, and Nova, dependent on $\theta_{13}, \delta$ and the mass hierarchy, there could be the first evidence for leptonic CP violation and knowledge of the mass hierarchy. However even in this optimistic scenario, the constraints on the value of $\delta$ will be poor and so an era of more precise experiments, starting around 2020, will be necessary

\section{The Precision Era}

By $\sim 2015$ there will be measurements of $\theta_{12}, \theta_{23}, \Delta_{12}^{2}, \Delta_{13}^{2}$, probably a measurement of $\theta_{13}$ and possibly the sign of $\Delta_{13}^{2}$ will be known. The aim of the precision era will be to measure $\delta$ accurately and refine all the parameters so that the information will be sufficiently good that theories/models which aim to predict neutrino parameters and particularly GUT and other theories which aim to relate the quark and lepton sectors can be rigorously tested. Six possible measurements for both neutrinos and antineutrinos are possible for long baseline oscillation experiments. Ideally all should be measured if the unitarity of the PMNS matrix and CPT conservation are to be tested. These are:

$\begin{array}{lll}v_{\mathrm{e}} \text { Beam } & v_{e} \rightarrow v_{e} & \text { 'disappearance' } \\ & v_{e} \rightarrow v_{\mu} & \text { 'golden channel' } \\ v_{e} \rightarrow v_{\tau e} & \text { 'silver channel' } \\ v_{\mu} \text { Beam } & v_{\mu} \rightarrow v_{e} & \text { 'platinum channel }\end{array}$




$$
\begin{array}{ll}
v_{\mu} \rightarrow v_{\mu} & \text { 'disappearance } \\
v_{\tau} \rightarrow v_{\tau e} & \text { 'silver channel' }
\end{array}
$$

The main factors that must be taken into account when planning such measurements are:

- Beam - Intensity, purity, divergence, energy, energy spread

- Detector - Size, energy resolution for $\mu$ 's and e's, detection threshold, magnetisable, threshold for sign determination if magnetised, pictorial (for $\tau$ 's and e's)

- Baseline - Range of L/E

- Backgrounds - Beam contamination, Time structure of beam

- Near Detector - Crucial for systematics, ideally same technology as far detector

- Systematics - Neutrino cross-sections, near/far comparison, Particle/antiparticle

- Cost

As the experiments become more complex costly choices will have to be made and it is the combination of these factors, plus the knowledge of $\theta_{13}$ which should determine the best approach for this era.

\subsection{Neutrino Source}

Future options for an intense neutrino source will no longer be limited to the conventional beam from $\pi$ decay but can also result from a beta decaying nucleus (beta beam) or from muon decay (neutrino factory) in a storage ring.

\subsubsection{Electron Neutrino Beams - the Golden Channel}

The golden channel, in which a muon is observed from an electron neutrino beam requires a $v_{\mathrm{e}}$ energy $>\sim 120 \mathrm{MeV}$ and so, although reactors are a prolific source of antielectron neutrinos, they are of such low energy that only disappearance experiments are possible. Thus, for the golden channel, either the beta beam or neutrino factory techniques must be employed.

The beta beam produces a very pure $v_{e}$ or $\overline{v_{e}}$ beam depending upon whether the ion decays via positron or electron emission and a wide range of detectors can be employed although energy resolution is important as the neutrinos have a broad energy spread. The neutrino factory will also produce a very pure $v_{e}$ or $\bar{v}_{e}$ beam with good intensity but from muon decay the $v_{e}$ or $\overline{v_{e}}$ are accompanied by a similar beam of ${\overline{v_{\mu}}}_{\text {or }} v_{\mu}$ which give rise to opposite sign leptons. Thus it is imperative for the neutrino factory that the detector must be magnetised in order to determine the muon sign and this complicates the choice. It is possible to have bunches of $\mu^{+}$and $\mu^{-}$from the neutrino factory to counter-rotate in the storage ring and so $v_{e}$ and $\bar{v}_{e}$ behaviour can be studied under virtually identical conditions. The neutrino energy spectrum is broad but it can go to higher energies than the beta beam.

\subsubsection{Muon Neutrino Beams - the platinum channel}

Muon neutrino beams can be generated either by the conventional approach from pion decay (superbeams) or using the neutrino factory. 
Superbeams are basically conventional neutrino beams where the power of the proton driver is > 1 MW. Neutrino or antineutrino beams are produced dependent upon the sign of the current in the magnetic horn. Most plans for superbeams, which will be capable of measuring $\delta$, assume a proton driver power of 2-4 MWs. Producing such a proton source and suitable target will not be easy but probably the main difficulty will depend upon whether the horn can survive the high number of wrong sign pions which are swept through it. Superbeams can either be onaxis or off-axis, the latter, as used in T2K and Nova, give a better tuned beam with smaller energy spread but ideally need two far detectors, preferably at first and second oscillation maxima to achieve full benefits. The wide band beam can cover both first and second oscillation maxima with one detector but not necessarily with the highest intensity at either.

Muon neutrino beams from a neutrino factory will have essentially the same properties as the $v_{\mathrm{e}}$ beams. However to take advantage of the platinum channel, determination of the sign of the electron is mandatory and a detection technique for this has yet to be proven.

\subsection{Detectors}

Detector development for precision neutrino experiments is just as crucial as the efforts to produce intense neutrino sources. The detectors need to be very large, have good energy resolution and, in the case of the neutrino factory, be magnetisable. Possibilties are:

Water Cerenkov. This is excellent at low energies when the interactions are quasi elastic, electrons and muons can be distinguished and their energy determined, it is almost certainly scalable, although costly, by an order of magnitude from Super-K. A large water Cerenkov also has the merit that it is the detector of choice for searching for proton decay. It is less good however as the neutrino energy increases and inelastic processes become important. There seems to be no hope in magnetising a large water Cerenkov.

Liquid Argon TPC. This is potentially the ideal detector but it has yet to be employed in an active experiment, It is a pictorial device and can potentially identify taus as well as electrons and muons, however the size required for such a cryogenic detector is a major engineering challenge and automatic identification of events and data reduction require solution. The technique is now being investigated in Europe, the USA and Japan and it is highly likely that it will be used in the future. It is also highly likely that it can be used for certain channels of proton decay. Magnetisation may be possible

Iron/Scintillator Sandwich (MIND). This would be a scaled up version of the MINOS detector. It is certainly feasible and magnetisable and currently forms the baseline detector for a neutrino factory complex. In the neutrino factory context an important feature is the low momentum threshold above which the muon sign can be determined as this is critical for the overall performance of the golden channel. A main difficulty with the MIND detector is poor electron measurement.

Totally Active Scintillating Detector (TASD). This is based on the philosophy of the planned Nova and Minerva detectors. Scintillator forms both the target and the detection device. It can have good energy resolution for both electrons and muons but needs to be extremely large due to the low density. Investigations about how it may be possible to magnetise such a huge 
volume are taking place and if a solution is found it could become the baseline for the neutrino factory programme. There is a possibility that it could also be used for proton decay.

Hybrid Emulsion Detector. Today the only method guaranteed to detect taus is the emulsion detector and so if the silver channels are seen to be important then an emulsion detector based on the OPERA design, as a second far detector, could be feasible. This is only really relevant for the neutrino factory which can provide a reasonable neutrino flux above the tau threshold and therefore it would need to be magnetised. Plans for such a detector (MECC) are under development.

Near Detector \& Beam Diagnostic devices. Characterising the beam close to its formation is important, critically so for the superbeam, and this depends on the near detector and the beam diagnostics. Ideally the near detector should employ the same technology as the far detector but this is not always feasible depending upon the constraints at the neutrino production site. As statistics increase the role of the near detector becomes increasingly important to reduce the systematic uncertainty.

A detailed summary of the possible detectors can be found in the ISS Detector Report[12]

\subsection{Possible Next Generation Superbeams}

Typical parameters for a future superbeam are:

- A proton driver in the range 2-4 MW

- Long Baselines with at least two L/E

- A very large far detector which is also a candidate for the search for proton decay

Plans exist for future superbeams in the three main regions although none are approved and finaproval apl is unlikely before more information on $\theta_{13}$ is available.

In Japan the plan is to boost the power of the JPARC neutrino beam to $\sim 4 \mathrm{MW}$ and currently three options for the detector are considered.

- T2HK. A very large detector Hyper-K (1 Mton water Cerenkov or 100 kton liquid Argon) close to the present Super-K detector.

- T2KK. To split the far detector and have half in Japan and half in Korea

- T2K to Okinoshima. Also to split the detector but put the second half on the island of Okinoshima between Japan and Korea. These are shown in Fig 8.

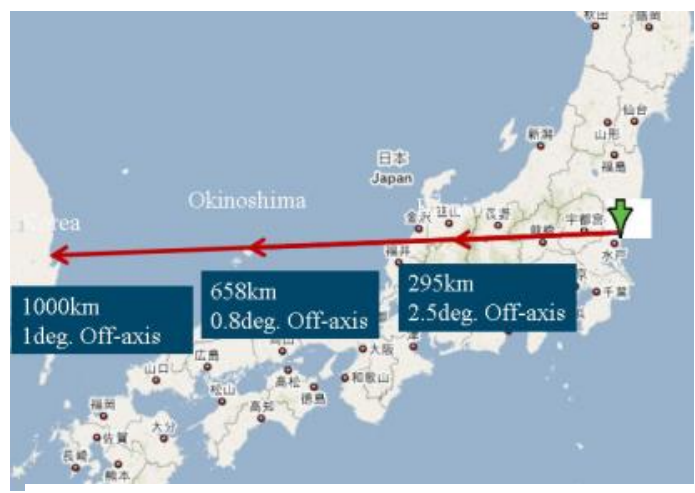

Fig 8. Japanese possibilities for future superbeams

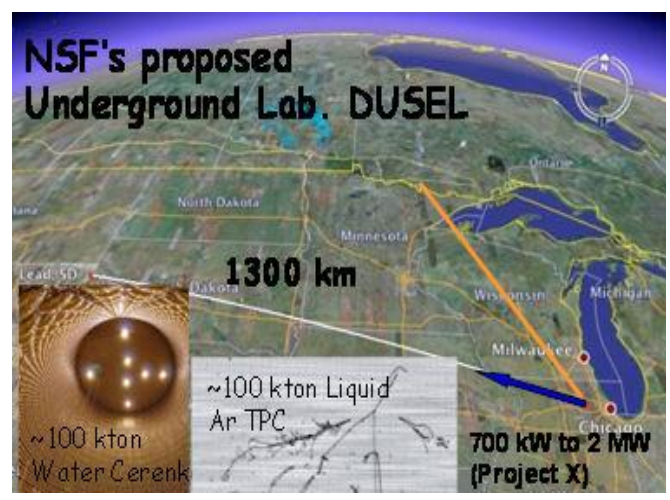

Fig 9. Possible future Superbeams from FNAL 
Plans in the US centre around Project X at FNAL which could deliver 2MW of protons at $120 \mathrm{GeV}$ to produce a wide band beam aimed at a large water Cerenkov or liquid argon detector at the future underground laboratory, DUSEL (Fig 9).

In Europe future superbeam plans are based on a $4 \mathrm{MW} \mathrm{SPL}$ at $4 \mathrm{GeV}$ producing a beam to a proposed large water Cerenkov, MEMPHYS, at a relatively short baseline in the Frejus tunnel.

\subsection{Beta Beams}

The beta beam concept originated with P Zucchelli[13] in 2002 and is investigated predominantly at CERN. Beta decaying ions are stored in a ring with straight sections so that as they decay they yield a pure neutrino beam, $v_{e}$ if the ion undergoes $\beta^{+}$decay, $\bar{v}_{e}$ if the ion undergoes $\beta^{-}$decay. The advantages are the purity of the beam, access to the golden channel and, as originally formulated, the ability to use much of the CERN infrastructure expected to be in place towards the end of the next decade. The main difficulty lies in producing enough ions at sufficiently high energy to obtain the desired interaction rate.

Choice of the ions is critical, ideally they should be light to obtain optimum Z/A and have a lifetime $\sim 1 \mathrm{sec}$ to be able to store them. The original scheme was to use ${ }^{6} \mathrm{He}$ for $\overline{v_{e}}$ and ${ }^{18} \mathrm{Ne}$ for $v_{e}$ and accelerate them to a $\gamma$ of 100, which is possible with the current CERN SPS. The energy spectrum of the neutrinos is broad extending from 0 to $\sim 2 \mathrm{Q} \gamma$, with $\mathrm{Q}$ the $\mathrm{Q}$ value of the decay leading to a mean energy of $\sim 350 \mathrm{MeV}$ for ${ }^{6} \mathrm{He}$ and $300 \mathrm{MeV}$ for ${ }^{18} \mathrm{Ne}$ at $\gamma=100$. This is rather low to be truly competitive with a neutrino factory. At present there are difficulties producing an adequate number of ${ }^{18} \mathrm{Ne}$ ions with the conventional ISOL method however calculations predict that, with a new direct production method, this difficulty can be overcome. It is under investigation. This overall scheme has been the subject of a design study as part of the EURISOL project and a report is expected in 2009 with details including estimated costs.

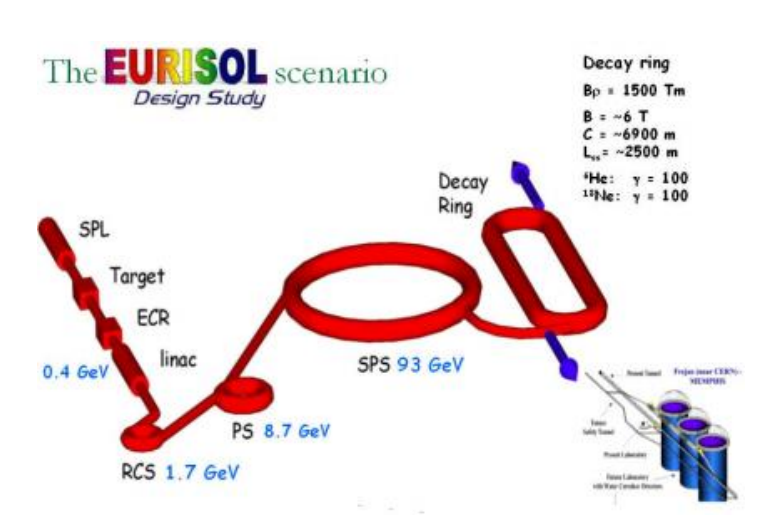

Fig 10. The beta beam scheme at CERN for the EURISOL design study.
The 'EURISOL' beta beam at CERN is shown in Fig 10.

The performance of the beta beam with $\mathrm{He}$ and $\mathrm{Ne}$ can be improved by accelerating the ions to a higher energy and a $\gamma$ of 350 has been investigated. At such a $\gamma$ the performance improves considerably and becomes competitive with a neutrino factory, however at CERN this would require a new SPS which is unlikely to happen before the mid 2020's and the problem of producing enough $\mathrm{Ne}$ ions remains. A $\gamma$ of 350 will require longer straight sections in the storage ring which will add to the cost and complexity.

To avoid the necessity to increase the $\gamma$ a new scheme has recently been proposed to use ions with significantly higher $\mathrm{Q}$ values. The proposal is to use ${ }^{8} \mathrm{Li}$ for $\bar{v}_{e}$ and ${ }^{8} \mathrm{~B}$ for $v_{e}$. These 
have $\mathrm{Q}$ values of 13 and $13.5 \mathrm{MeV}$ respectively, approximately 4 times those for ${ }^{6} \mathrm{He}$ and ${ }^{18} \mathrm{Ne}$ and so at $\gamma=100$ the performance is comparable to these ions at $\gamma=350$. However it is considered impracticable to produce adequate numbers of these ions by conventional means and an approach involving an accumulation ring has been proposed. This is being investigated in the present EuroNu FP7 project but for ${ }^{8} \mathrm{~B}$ in particular it looks quite demanding.

If one assumes the standard 3 generation PMNS picture the golden channel, using a range of $\mathrm{L} / \mathrm{E}$, is by far the most efficient process to measure the parameters due to the ease of identifying the final state muon and determining its energy. In this scenario a reasonably high energy beta beam is very attractive if it can be achieved at an acceptable cost. The major advantages over the neutrino factory being the fact that the associated detector does not need to be magnetised and it may be possible to use a substantial part of the planned CERN infrastructure and so reduce the cost.

\section{The Neutrino Factory}

The neutrino factory, producing neutrinos result from the decay of muons in a storage ring is generally considered the ultimate device currently realisable to produce intense pure neutrino beams. A muon however yields both a muon neutrino and electron antineutrino or vice-versa and so sign determination in the detector becomes mandatory to determine if the interaction was initiated by a neutrino or antineutrino. Due to the short muon lifetime the neutrino factory is

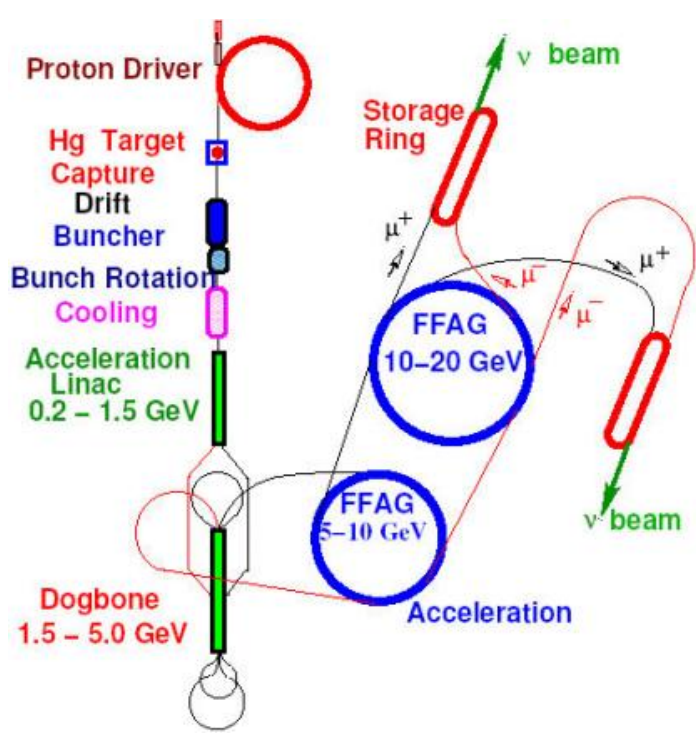

Fig 11. The basline design for the IDS Neutrino Factory Study complicated but it has been the subject of numerous studies, the last one being the International Scoping Study (ISS) which took place between 2005 and 2007 and which forms the baseline for current R\&D in the neutrino factory area. This is now coordinated by the International Design Study (IDS).

The baseline design resulting from the ISS study[14] is shown in Fig 11. The sequence of operations is dictated by the short muon lifetime which rules out ramping magnets and conventional cooling techniques. A proton driver giving $4 \mathrm{MW}$ at $5-15 \mathrm{Gev}$ impacts a target producing pions.

Unlike the superbeam the target sits in a solenoidal field and so both signs of pions are captured. In the front end the decay muons are bunched with interleaved opposite sign bunches and phase rotated to a uniform energy before entering the cooling channel where ionisation cooling reduces the emittance prior to the acceleration stages. Acceleration is accomplished, first with a recirculating linac and then one or two FFAG's. Two racetrack decay rings are assumed so that two very different baselines can be used and counter rotating bunches of opposite sign muons in each ring enable neutrino 
and antineutrino interactions to be investigated. Two major problems are the target and the cooling. The baseline for the target is a mercury jet and a proof of principle for this has been shown by the MERIT experiment at CERN although many technical problems remain to be
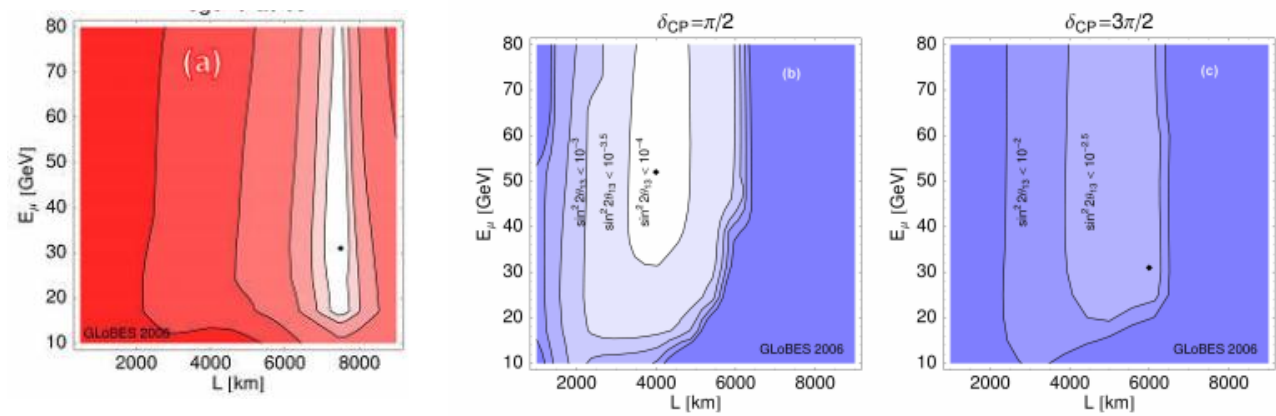

Fig 12. Optimisation of the muon storage ring energy and the baseline for a neutirno factory, (a) for $\theta_{13}$, (b) for $\delta$, with $\delta=\pi / 2$, (c) for $\delta$, with $\delta=3 \pi / 2$

solved. Ionisation cooling appears to be the only technique to reduce the emittance but as it has it has never been demonstrated in practice it is now the focus of the MICE experiment at RAL which will produce results by 2012 .

The performance of a neutrino factory was extensively investigated by the ISS physics group[15]. An important parameter is the energy of the stored muon beam. High energy is desirable and earlier plans had assumed an energy of $50 \mathrm{GeV}$, however the cost depends upon the number of accelerating stages and the study showed that an energy around $20 \mathrm{GeV}$ was satisfactory. For $\theta_{13}$ the maximum sensitivity, with effectively no degeneracies, occurs at $\sim 7500$ $\mathrm{km}$ and this is referred to as the magic baseline, whilst for $\delta$, at $20 \mathrm{GeV}$, there is a broad minimum from around 3000 to $5000 \mathrm{~km}$ depending upon the value of $\delta$, shown Fig 12 . Consequently the IDS baseline has MIND-type detectors at 3500 and $7500 \mathrm{~km}$.

Such long baselines also mean that the neutrino factory is ideal to determine the mass hierarchy should $\theta_{13}$ be very small.

The planned new underground laboratory, DUSEL, in the USA has generated much recent interest in a low energy neutrino factory $(\sim 4 \mathrm{GeV}$ in the muon storage ring) at FNAL as part of Project $\mathrm{X}$, possibly as a precursor to a higher energy version. This is currently under serious investigation with the emphasis on the detector as it is the low energy threshold for the muon
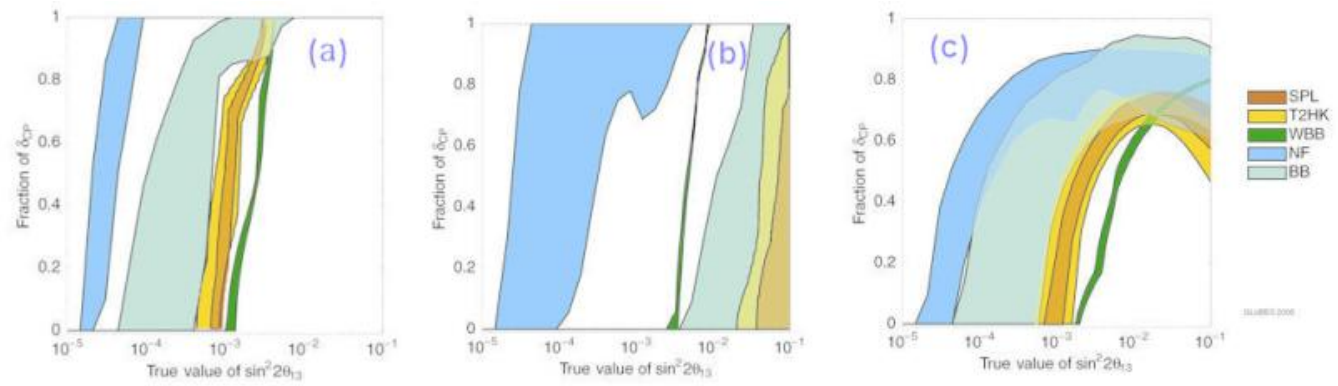

Fig 13. Comparison of the expected performance as a function of $\sin ^{2} 2 \theta_{13}$ for (a) $\theta_{13}$, (b) the mass hierarchy (c) $\delta$. SPL, T3HK and WBB are possible superbeams in Europw, Japan and the USA, NF corresponds to the neutrino factory and BB the beta beam 
sign determination in the golden channel which will dictate the performance. This almost certainly means that the MIND type of detector, which was used for the above studies, is unsuitable, however a TASD type of detector, if magnetisable, could satisfy the requirements and lead to an effective facility.

\subsection{A performance Comparison}

In the ISS physics report a comparison was made of the sensitivity for $\theta_{13}, \mathrm{CP}$ violation and the mass hierarchy as a function of $\sin ^{2} 2 \theta_{13}$ that can be expected. Three potential superbeams, T2HK, a wideband beam from FNAL to DUSEL ('WBB') and a beam originating at CERN to a Frejus detector ('SPL'), were examined along with beta beams with $\gamma=100$ and 350 and the neutrino factory with conservative and optimistic performance of the detectors. Some of the results are shown in Fig 13 In all cases the neutrino factory outperforms the other facilities except for high $\theta_{13}$, i.e. just below the Chooz limit, when all show a similar behaviour for the detection of CP violation. Full details can be found in the ISS physics report.

\section{Summary}

The Lepton Sector has shown the first sign of BSM physics and may hold the key to the Flavour problem, CP violation and a viable GUT, however progress will need precision measurements of the oscillation parameters, at least comparable with those determined for the CKM matrix. This will demand major construction beyond T2K and Nova to produce intense neutrino beams with very large detectors. Realistic proposals exist for both beams \& detectors but they require significant R\&D to show viability. Some of this is taking place now. By $2012-$ 2014 there should be enough information, R\&D results, cost estmates and crucially the value of $\theta_{13}$ to decide the best approach for a precision era experiment which could take data by 2020-25. It is vital also to continue to refine and extend efforts to observe and measure neutrinoless double beta decay and determine the absolute mass.

\section{References}

[1] Ch Kraus et al, Final results from phase II of the Mainz neutrino mass search in tritium beta decay, Eur.Phys.J.C40:447-468,2005 [hep-ex/0412056]

[2] V.M. Lobashev et al. Direct search for mass of neutrino and anomaly in the tritium beta spectrum. Phys.Lett.B460:227-235,1999

[3] Y. Fukuda et al., Evidence for oscillation of atmospheric neutrinos. Phys.Rev.Lett.81:15621567,1998 [hep-ex/9807003]

[4] M.H. Ahn et al. Measurement of Neutrino Oscillation by the K2K Experiment. Phys.Rev.D74:072003,2006 [hep-ex/0606032 ] 
[5] P. Adamson et al. Measurement of Neutrino Oscillations with the MINOS Detectors in the NuMI Beam.Phys.Rev.Lett.101:131802,2008. [arXiv:0806.2237]

[6] ${ }^{1}$ Q.R. Ahmad et al. Direct evidence for neutrino flavor transformation from neutral current interactions in the Sudbury Neutrino Observatory. Phys.Rev.Lett.89:011301,2002.

nucl-ex/0204008

[7] ${ }^{1}$ S. Abe et al. Precision Measurement of Neutrino Oscillation Parameters with KamLAND. Phys.Rev.Lett.100:221803,2008. [arXiv:0801.4589]

[8] ${ }^{1}$ A. Aguilar et al. Evidence for neutrino oscillations from the observation of anti-neutrino(electron) appearance in a anti-neutrino(muon) beam. Phys.Rev.D64:112007,2001. [hep-ex/0104049]

[9] ${ }^{1}$ H.V. Klapdor-Kleingrothaus, I.V. Krivosheina, A. Dietz, O. Chkvorets, Search for neutrinoless double beta decay with enriched Ge-76 in Gran Sasso 1990-2003. Phys.Lett.B586:198-212,2004. [hep-ph/0404088]

[10] ${ }^{1}$ M. Apollonio et al. Limits on neutrino oscillations from the CHOOZ experiment. Phys.Lett.B466:415-430,1999. [hep-ex/9907037]

[11] ${ }^{1}$ P.F. Harrison, D.H. Perkins, W.G. Scott, Tri-bimaximal mixing and the neutrino oscillation data. Phys.Lett.B530:167,2002. [hep-ph/0202074]

[12] ${ }^{1}$ The ISS Detector Working Group, Detectors and flux instrumentation for future neutrino facilities. JINST 4 T05001 2009

[13] ${ }^{1} \mathrm{P}$. Zucchelli, A novel concept for a anti-nu/e / nu/e neutrino factory: The beta beam. Phys.Lett.B532:166-172,2002

[14] ${ }^{1}$ The ISS Accelerator Working Group Accelerator design concept for future neutrino facilities JINST 4 P07001 2009

[15] ${ }^{1}$ The ISS Physics Working Group, Physics at a Future Neutrino Factory and super-beam. To be published in Rep Prog Phys. 